University of Nebraska - Lincoln

DigitalCommons@University of Nebraska - Lincoln

Faculty Publications, Department of Psychology

Psychology, Department of

2003

Comparison of Implicit and Explicit Learning Processes in a

Probabilistic Task

Thomas D. Green

Elon University, greent@elon.edu

John H. Flowers

University of Nebraska-Lincoln, jflowers1@unl.edu

Follow this and additional works at: https://digitalcommons.unl.edu/psychfacpub

Part of the Psychiatry and Psychology Commons

Green, Thomas D. and Flowers, John H., "Comparison of Implicit and Explicit Learning Processes in a Probabilistic Task" (2003). Faculty Publications, Department of Psychology. 449.

https://digitalcommons.unl.edu/psychfacpub/449

This Article is brought to you for free and open access by the Psychology, Department of at DigitalCommons@University of Nebraska - Lincoln. It has been accepted for inclusion in Faculty Publications, Department of Psychology by an authorized administrator of DigitalCommons@University of Nebraska - Lincoln. 
Published in Perceptual and Motor Skills 97 (2003), pp. 299-314. Copyright (C) 2003 Perceptual and Motor Skills; published by Ammons Scientific Ltd.. Used by permission.

Accepted for publication July 14, 2003.

\title{
Comparison of Implicit and Explicit Learning Processes in a Probabilistic Task
}

\author{
Thomas D. Green, Elon University \\ John H. Flowers, University of Nebraska-Lincoln \\ Corresponding author - Thomas D. Green, Department of Psychology, \\ Elon University, Elon, NC 27244; email greent@elon.edu
}

\begin{abstract}
This experiment compared the performance with explicit (rule-application and rule-discovery) and implicit (nonrule-instructed) learning approaches on the performance of a probabilistic video game task requiring fine motor control. The task required visual tracking of a small ball of light and "catching" it by means of joystick manipulation. A general pattern of improvement with practice occurred for all conditions. All conditions showed use of predictive relations among stimulus events. However, task performance of the ruleapplication and rule-discovery conditions were inferior to the nonrule-instructed implicit condition, particularly during the early phases of rule acquisition and application. This pattern strongly suggests substantial performance costs associated with attempting to discover or apply probabilistic rules. Decrements are likely due to increased cognitive demands associated with attempting to remember and strategically apply provided probability rules or attempting to discover and apply potentially important and useful probability information from a complex visual display.
\end{abstract}

Many real-world tasks as well as experimental laboratory tasks involve predictive relationships between stimulus events. Important issues surrounding these tasks concern whether individuals can learn to use such predictive relationships, and if one should be informed of the existence or the nature of such relationships in an effort to facilitate performance. The following realworld scenario nicely illustrates these issues. Imagine that you are a baseball batting coach, and you are aware of a specific predictive relationship involved in the pitching movements of the opposing lead pitcher for an upcoming contest. It seems that three out of four times, the pitcher makes a noticeable outward movement of the right elbow during the pitching move just prior to a right to left curve pitch. Knowing this information, would you tell your batters that a probability relationship exists, the exact nature of the probability relationship, e.g., 3 out of 4 , or would you tell them nothing?

The general issues raised in this scenario are central to the field of implicit and explicit learning research, which addresses basic research tasks such as visual target search and pattern sequence learning, as well as more applied 
types of tasks such as learning grammar. Implicit learning has been defined as the process involved in the acquisition of abstract, unconscious knowledge about rule-governed covariations present in one's stimulus environment, without conscious effort (Reber, 1989). In contrast, explicit learning has been characterized as a process similar to conscious problem-solving used for discovering and controlling task variables (Mathews, Buss, Stanley, BlanchardFields, Cho, \& Bruhan, 1989), which gives rise to conscious, concrete knowledge of the regularities in one's stimulus environment (Reber, 1989).

Several studies have investigated individuals' implicit acquisition and use of probability relationships, e.g., predictive covariations, rule-governed complexities, among stimulus events. A key characteristic of this research is that subjects were not provided verbal instructions concerning the existence or nature of the probability relationships. This research has addressed a variety of reaction time-type tasks involving social cognition (Lewicki, 1986), visual target search (Lewicki, Czyzewska, \& Hoffman, 1987; Lewicki, Hill, \& Bizot, 1988; Stadler, 1989), pattern sequence learning (Nissen \& Bullemer, 1987), and event sequence learning (Reber \& Millward, 1971; Millward \& Reber, 1972). Also, complex rule-governed tasks involved in simulated production systems (Broadbent \& Aston, 1978; Berry \& Broadbent, 1984; Broadbent, FitzGerald, \& Broadbent, 1986), and learning artificial grammar (Reber, 1967; Mathews, et al., 1989) have been addressed, as well as visual-motor continuous tracking tasks (Firth \& Lang, 1979; Notterman \& Tufano, 1980; Mather \& Putchat, 1983). The results across this varied array of tasks have, in general, shown subjects implicitly, unconsciously acquire and subsequently use knowledge of predictive relationships between stimulus events.

Other studies have investigated the use of specific probability information provided through explicit instruction (explicit rule-application). Here, subjects were not only told about the existence of a statistical contingency but were also told specifically what the contingency was and were encouraged to use it. Typical examples of such tasks are reaction time-type tasks involving lexical decisions (Neely, 1977), character classification (Taylor, 1977; Flowers, Nelson, Carson, \& Larsen, 1984; Flowers, Reed, \& Green, 1991), and detection (Posner \& Snyder, 1975). The results associated with these tasks have, in general, shown facilitation of performance as a result of using probability information.

Unlike the studies directed specifically toward implicit learning or explicit rule application, only a few studies have compared the utilization of implicit versus explicit processes. These comparison studies can be categorized as those contrasting implicit learning to explicit rule-application learning and those comparing implicit learning to explicit rule-discovery learning.

In studies involving comparisons between explicit rule discovery and implicit learning, the implicit learning group typically receives no instructions as to specific probability relationships inherent in the task, while the rule discov- 
ery group is informed about the existence of probability relationships among stimulus events so as to encourage the discovery and application of the specific relationships. The tasks have involved learning artificial grammar (Reber, 1976; Brooks, 1978; Millward, 1981; Abrams, 1987), and paired-associate learning (Brooks, 1978). The results of these studies have shown both no difference between the noninstructed implicit group and the instructed explicit rule-discovery group (Millward, 1981; Abrams, 1987) and lower performance for the explicit rule-discovery group (Reber, 1976; Brooks, 1978).

In studies comparing explicit rule-application learning to implicit learning, the rule-application group is verbally instructed as to the exact nature (degree) of the probability relationship, while the implicit group, as noted above, receives no instructions as to specific probability relationships. Results from two studies of event-sequence learning (Reber, 1966; Reber \& Millward, 1968) yielded no effect of providing subjects with concrete knowledge of event probabilities. However, Green and Flowers (1991), in comparing an implicit group to a rule-application group, found that explicit verbal instructions concerning probability relationships among key stimulus features led to poorer overall performance for a probabilistic continuous fine motor task.

From these studies, it is clear that subjects can implicitly learn probability information and can apply explicitly provided probability information. However, the findings concerning the utility of providing instructions about the existence (rule discovery) or specific nature of probability relationships (rule application) as compared to implicit learning are mixed and less clear. It appears that, in contrast to what common sense might suggest, providing subjects with explicit information about the existence of probability relationships, or providing subjects with explicit information as to the nature of the probability relationships is either not beneficial or potentially detrimental to task performance.

It has been suggested that performance decrements observed in studies of rule discovery (Reber, 1976; Brooks, 1978) and the rule-application (Green \& Flowers, 1991) comparative studies are due to differential demands placed upon subjects' cognitive resources which result in performance costs for the explicitly instructed conditions (Green \& Flowers, 1991). In other words, effort in trying to discovery and use, or remember and apply rules detract from cognitive resources needed for optimally performing the tasks. Further, it has been suggested that the degree of disruption is likely dependent on the type of instruction involved (Green \& Flowers, 1991). However, there are no studies which specifically contrast explicit rule discovery to explicit rule application.

Because the findings from studies which involve a comparison between implicit versus explicit learning are mixed and no direct comparison of rulediscovery and rule-application approaches has been made, the general pur- 
pose of our study was to clarify whether explicit instructions are detrimental to skilled performance and to compare the relative quantitative and qualitative effects on performance of rule-application and rule-discovery procedures. In doing so, our study compared the performance effect of explicit rule-application instruction (e.g., instructions that Event " $\mathrm{A}$ " predicts Event "B" a given percentage of the time), explicit rule-discovery instruction (e.g., instructions that a predictive rule exists), and implicit learning (no instruction) on participants' ability to learn and utilize correlations among visual events in a task that required the manipulation of a computer joystick to "catch" a ball of light as it "dropped" across a computer screen. Like many real-world tasks, the task involved predictive relationships between key stimulus features or characteristics, and incorporated a blend of discrete events and continuous visual and motor activity.

We hypothesized that participants in all conditions would demonstrate general improvement with practice and would show application or use of the probabilistic relationships between stimulus features. It was also predicted that various processing loads associated with different conditions would lead to differing performances. In general, we predicted that higher processing loads and thus poorer performances would be experienced by the Rule-discovery and Rule-application conditions as compared to the Implicit (no instruction) condition. More specifically, it was predicted that attempting to discover and utilize probability rules would lead to the largest processing load and therefore the worst overall performance for the Rule-discovery condition. Similarly, it was predicted that attempting to remember and utilize probability information would also lead to a substantial, although relatively lower, processing load and therefore a lower performance difference between the Implicit and the Rule-application conditions.

We also predicted that differing attentional demands required for discovering and applying probability rules, or in applying explicitly provided rules would likely lead to stylistic differences in the patterns of joystick movements across conditions. Specifically, it was predicted that the Rule-discovery condition would show reduced joystick movement during the entire movement of pathways, particularly during early sessions, as a result of an increased overall attentional focus associated with discovering rules. The Rule-application condition was predicted to show reduced joystick movement during the period of time that subjects were overtly looking for a critical predictive feature of a ball's pathway, followed by increased movement following detection of such a feature. The Implicit (no instruction) condition was predicted to show moderate activity across the full length of the pathways as a result of attention being focused more on simply doing the task instead of discovering or applying probabilistic rules. 


\section{Method}

\section{Participants}

Twenty-seven students (12 men and 15 women) volunteered to participate from various undergraduate psychology courses. Physical criteria included visual acuity corrected to $20 / 20$ and physical ability to operate manually a personal computer with a joystick attachment. An additional criterion was that a participant's native language be English. No other special characteristics were required.

\section{Apparatus}

The apparatus consisted of four identical personal computer workstations located in a well-illuminated laboratory room. Each workstation included a desk and chair, with an Apple IIe computer, an Apple IIe monitor (p31 phosphor video screen), and a Suncom Tac1+ analog joystick placed on the desk.

\section{Procedure}

Participants engaged in a catching task (similar to a video game) for five 1-hr. daily sessions. At the beginning of the first session, the experimenter showed participants the computer-operation procedures and directed the participants to read carefully specific instructions presented on the computer screen. As a reminder, the instructions were presented on the computer screen at the beginning of each of the remaining four sessions. Instructions are described in detail below in the section labeled "instructional conditions and feature correlations."

During each daily session, participants were presented with six blocks of 32 task trials. Each trial involved visually tracking a small dot or "ball" of light which appeared on the computer screen as it moved from the top to the bottom of the screen and attempting to "catch" the ball by manipulating the joystick that controlled a cursor at the bottom of the screen. The balls followed curved pathways that included features that predicted the balls' final movement with 75\% accuracy. Pathways are described in detail below in the section labeled "stimulus conditions." Participants "caught" the ball when the cursor intersected the final position of the ball. The cursor was five pixels wide, and the ball consisted of a single pixel. As performance feedback, participants were assigned 3 "points" for catching the ball in the middle or third pixel of the cursor, 2 points for catching it on the second or fourth pixel, and 1 point for catching it on either the first or fifth pixel. Trials on which a catch occurred were rewarded by a visual display of the number of points (1-3) assigned for that catch. The point total for each block of 32 trials was displayed on the screen following completion of the block. Point total data 
1

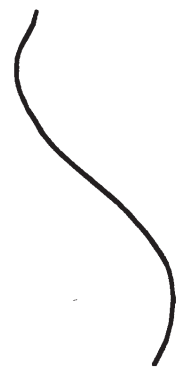

3

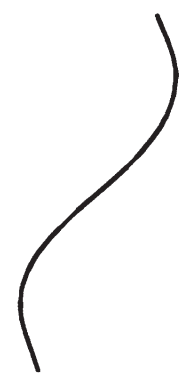

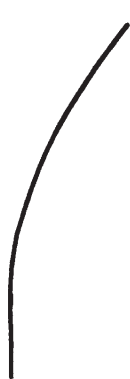

2

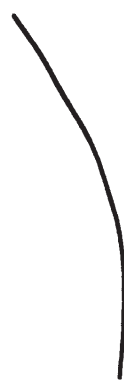

4

Figure 1. Examples of four prototype pathways

were only used for participants' feedback and motivation. The actual deviation of the ball's position from the center pixel was used as the primary dependent measure as described in detail below in the section labeled "dependent variables."

At the end of the fifth session, participants responded to a brief exit questionnaire designed to measure participants' explicit awareness of predictive features of the pathways, and participants' explicit perception of the predictive relationships between pathway features. The exit questionnaire is discussed in greater detail below in the section labeled "exit questionnaire."

Stimulus conditions. - During each of these five 1-hr. sessions, participants were presented five blocks of 32 stimulus trials. Therefore, each participant completed 180 trials during each session for a total of 800 trials during the experiment.

Each trial consisted of a 2.5 -sec. display (1.50 video frames) sequence in which the ball moved from the top edge of the video display to an imaginary "end line" at the bottom of the screen. The path followed by the ball consisted of one of four prototype pathways. The prototype pathways consisted of two single curve pathways and two "S" curve pathways as shown in Figure 1. 
Each prototype pathway varied with respect to the presence or absence of two distinct features. The first of these features was a "glitch" consisting of a five-frame excursion to the right followed by a five-frame return to the normal pathway. The glitch provided the appearance of a "bump" or "jiggle" in an otherwise smooth pathway. When present, the glitch appeared in Frames 50 to 69 of the display sequence or about one-third of the distance from the top of the screen to the goal line. The second feature was a fade which consisted of a sharp "break" or "curve" to the right during the final 18 frames or $300 \mathrm{msec}$. of the trial. When present, the fade resulted in a 9-pixel displacement to the right of the final ball position of trials in which no break occurred. The starting position of individual pathways was randomly varied within a restricted range at the top of the screen. The range was restricted to ensure that the final position of the ball would be in an area which would allow a catch.

Presentation of the four prototype pathways under the possible combination of the two binary (presence vs. absence) features resulted in 16 possible pathways. Figure 2 shows examples of the four possible applications of the Glitch and Fade features on the third prototype pathway shown in Figure 1.

Instructional conditions and feature correlations. - All participants in the three conditions (Rule-application, Rule-discovery, and Implicit conditions) were presented trial sequences for which the Glitch and Fade features were correlated. Across these trials, the Glitch feature was followed with a Fade feature $75 \%$ of the time; and, when the Glitch feature was not present, the Fade feature was absent $75 \%$ of the time. Thus, the correlation between the presence of Glitches and Fades could potentially be used by participants as a cue to prepare for the rightward displacement of the ball in the final few frames. Similarly, the correlation between the absence of Glitches and Fades could be potentially used by participants.

Participants in the Rule-application condition ( 4 men and 5 women) were explicitly instructed via computer video display that on $75 \%$ of the trials, if the ball jiggles (makes a momentary excursion from its pathway) during the top third of the screen, then it will fade or break to the right during the last portion of its path. Further, subjects were instructed that if no jiggle occurs, then the ball will not break on $75 \%$ of the trials. Along with the video instructions, the Rule-application group was provided with a video display of the four variations of the third prototype pathway shown in Figure 2.

Participants in the Rule-discovery condition (4 men and 5 women) received the same stimulus sequences as those in the Rule-application condition and were likewise shown the four variations of the prototypic pathway. However, these participants were only provided the instructions concerning the existence of predictive relationships somewhere in the path- 
No Glitch - No Fade

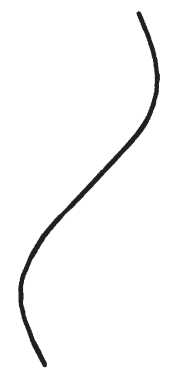

Glitch - No Fade

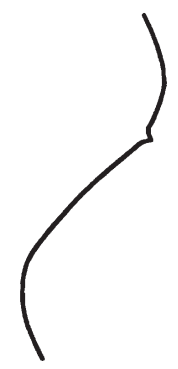

No Glitch - With Fade

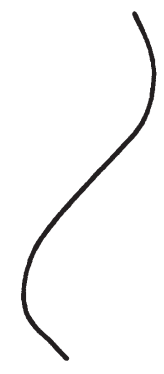

Glitch - With Fade

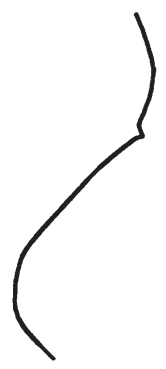

Figure 2. Example of Prototype Pathway 3 with four feature applications

ways. Therefore, they had the opportunity to discover and use the GlitchFade contingency.

Participants in the Implicit condition (4 men and 5 women) saw the same stimulus sequences as those in the Rule-application and Rule-discovery conditions and were similarly shown the four variations of the prototypic pathway. However, these participants were not given instructions about the existence or nature of the statistical relationships between the Glitch and Fade features. Thus, they had the opportunity to learn the Glitch-Fade contingency solely through experience.

Dependent variables. - Two different dependent variables were of interest, Error of catch and Joystick movement. Error of catch was measured as the absolute deviation of the ball from the center of the paddle in numbers of pixels on the final video frame of each trial, when the ball reached the goal line. Joystick movement was measured as the standard deviation of paddle position values (the horizontal pixel location of the third dot of the paddle cursor) 
during 30 frame blocks on the video display. Thus, Joystick movement was computed for five consecutive blocks of 30 successive video frames on each trial which resulted in five Joystick movement scores for each trial. The purpose of using this variable was to provide a performance measure that characterized the tracking style. The data used for computing these two dependent variables were obtained by recording the joystick value (cursor position) and ball position on each of the 150 video frames on each trial.

\section{Experimental Design and Statistical Analysis}

Experimental design. - Two experimental designs were employed. The first design concerned with instruction effects on error score was a $3 \times 5 \times 4 \times 2 \times$ 2 mixed factorial model in which the first factor was a between-subjects factor, with the remaining factors being within-subjects factors. The first factor was instructional condition (Rule-application, Rule-discovery, Implicit). The remaining factors were session (1-5), pathway type (4 levels), Glitch feature (present, absent), and Fade feature (present, absent).

The second design, concerned with instruction effects on the Joystick movement score, was the same as the above design with the addition of another within-subjects factor, display segment (5 levels), reflecting the times at which the measures were taken during each trial as the target moved down the computer screen.

Statistical analysis. - Prior to analyses involving the Error of catch score and Joystick movement, data were aggregated. Data for each participant from the 180 trials within each session were blocked on pathway type and the presence or absence of the Glitch and Fade features, and then scores were aggregated across trials within the blocking variables so as to yield mean Error of catch and mean Joystick movement scores for each of the four feature combinations for each of the four pathway types within each session. Based on the two above experimental designs, then data were analyzed with analyses of variance for the mixed factorial models.

\section{Exit Questionnaire}

To ascertain participants' explicit knowledge or perception of the existence of the Glitch and Fade features, as well as their perception of the existence and magnitude of the predictive relationship between the features, a six-item questionnaire was employed. The first two items assessed if participants noticed a glitch or a fade during the task. Items 3 and 4 assessed whether participants perceived a correlation between the presence of the features and requested a percentage rating of the magnitude of the perceived predictive relationship. The last two items assessed whether participants perceived a correlation between the absence of the features and requested a percentage 
rating of the magnitude of the perceived predictive relationship. Statistical comparisons were made between the instructional conditions via two-sample chi square analyses for yes/no categorical response items and independentgroups analyses of variance for percentage rating items.

\section{Results}

\section{Error Score}

Error of catch scores, presented in Table 1, indicate the effect of practice on the error of catch scores across sessions for the Rule-application, Rule-discovery, and Implicit conditions. Table 2 depicts the effect of the Glitch and Fade features on the error of catch scores for each condition.

Session and instructional effects. - As shown in Table 1 and supported by a statistically significant main effect for practice session $\left(F_{4,96}=22.19, p<.01\right.$, $\left.\eta^{2}=.48\right)$, a substantial pattern of improvement with practice was present across conditions. The pattern of improvement, however, was most pronounced in the Rule-application and Rule-discovery groups with less improvement in the Implicit group. Although greater improvement was shown by the Rule-application and Rule-discovery groups, the different patterns of improvement suggest substantial performance costs in early sessions under these conditions, whereas in the Implicit condition participants benefited in early sessions from the lack of explicit instruction. The differential improvement is reflected in a significant interaction between Practice Session and Instructional Condition $\left(F_{8,96}=3.53, p<.01, \eta^{2}=.23\right)$.

In addition to mediating the effect of practice session, instructional condition exerted a main effect on performance which was significant at a mar-

Table 1. Mean error score (in pixels) as a function of session and instructional condition

\begin{tabular}{lcccccc}
\hline \multirow{2}{*}{ Instructional Condition } & \multicolumn{6}{c}{ Session } \\
\cline { 2 - 7 } & 1 & 2 & 3 & 4 & 5 & Marginals \\
\hline Implicit & & & & & & \\
$\quad M$ & 5.73 & 5.08 & 5.44 & 5.08 & 5.19 & 5.30 \\
$\quad S D$ & 4.41 & 2.99 & 3.09 & 2.97 & 3.85 & 3.51 \\
Rule-discovery & & & & & & \\
$\quad M$ & 8.39 & 6.89 & 6.48 & 5.60 & 5.54 & 6.58 \\
$\quad S D$ & 5.26 & 4.22 & 3.85 & 3.41 & 3.27 & 4.19 \\
Rule-application & & & & & & \\
$\quad M$ & 7.47 & 5.96 & 5.23 & 5.40 & 4.83 & 5.78 \\
$\quad S D$ & 6.30 & 3.62 & 3.70 & 4.79 & 3.38 & 4.57 \\
Overall Marginals & & & & & & \\
$\quad M$ & 7.20 & 5.98 & 5.72 & 5.36 & 5.18 & \\
$\quad S D$ & 5.48 & 3.71 & 3.60 & 3.80 & 3.52 & \\
\hline
\end{tabular}


ginal alpha level $\left(F_{2,24}=3.10, p<.06, \eta^{2}=.21\right)$. As shown in Table 1 , overall error of catch scores of the Implicit condition were lower than in the Rule-application condition, which in turn were lower than in the Rule-discovery condition. However, as shown, this difference was most pronounced in the early sessions, which again reflects the performance costs associated with the Ruleapplication and Rule-discovery conditions and the interaction between Practice Session and Instructional Condition.

Pathway feature effects. - As shown in Table 2, responses to pathways ending in a fade resulted in substantially higher error of catch scores as compared to pathways not ending in a Fade, thereby reflecting the overall greater difficulty of these pathways. This pattern of data resulted from a significant main effect $\left(F_{4,96}=22.19, p<.01, \eta^{2}=.95\right)$ of the Fade feature (presence vs. absence). All conditions showed use of the predictive relationships between the Glitch and Fade features as evidenced by a different pattern of costs and benefits associated with different combinations of these features. A pattern of costs and benefits can be seen across conditions in that error of catch scores were much lower for pathways that incorporated a Fade if a Glitch was present compared to when a Glitch was not present. Similarly, a pattern of costs and benefits can be seen across conditions in that error of catch scores were lower for pathways that did not include a Fade if a Glitch was not present, as occurred when a Glitch was present. This pattern of data resulted from a significant interaction between Glitch and Fade features $\left(F_{1,24}=104.74, p<.01\right.$, $\left.\eta^{2}=.81\right)$. Further, the pattern of costs and benefits was stable across practice sessions, as indicated by the lack of a significant interaction among Glitch, Fade, and Session $(F<1.00)$. However, it should be noted that there was a significant interaction for Glitch $\times$ Fade $\times$ Instruction $\left(F_{2,24}=3.63, p<.05\right)$. Simple effects analyses indicated that the interaction of Glitch $\times$ Fade was significantly present in all conditions: Implicit $\left(F_{1,24}=32.05, p<.01\right)$, Rule-application $\left(F_{1,24}=34.84, p<.01\right)$, and Rule-discovery $\left(F_{1,24}=42.60, p<.01\right)$. With respect to the three-way interaction, inspection of Table 2 shows that, although sig-

Table 2. Mean error score (in pixels) as a function of feature application for implicit, rule-discovery, and rule-application conditions

\begin{tabular}{|c|c|c|c|c|c|c|}
\hline \multirow[t]{3}{*}{ Fade } & \multirow{2}{*}{\multicolumn{2}{|c|}{$\frac{\text { Implicit }}{\text { Glitch }}$}} & \multirow{2}{*}{\multicolumn{2}{|c|}{$\frac{\text { Rule-discovery }}{\text { Glitch }}$}} & \multirow{2}{*}{\multicolumn{2}{|c|}{$\frac{\text { Rule-application }}{\text { Glitch }}$}} \\
\hline & & & & & & \\
\hline & Present & Absent & Present & Absent & Present & Absent \\
\hline \multicolumn{7}{|c|}{ Present } \\
\hline $\mathrm{M}$ & 6.17 & 7.48 & 7.43 & 9.06 & 6.43 & 8.72 \\
\hline SD & 2.19 & 4.67 & 3.09 & 4.84 & 2.52 & 6.74 \\
\hline \multicolumn{7}{|c|}{ Absent } \\
\hline M & 3.96 & 3.60 & 5.28 & 4.55 & 4.46 & 3.50 \\
\hline SD & 2.29 & 2.71 & 3.49 & 3.53 & 2.87 & 2.82 \\
\hline
\end{tabular}


nificantly present in each condition, the interaction of Glitch $\times$ Fade and associated costs and benefits were more pronounced in the Rule-application and Rule-discovery conditions. This pattern not only shows that all participants in all conditions utilized the predictive relationships between the predictive features but also indicates the pattern of costs and benefits to be greater for the explicitly instructed conditions. It should be further noted that the interaction for Glitch $\times$ Fade $\times$ Instruction was stable across practice sessions, as indicated by the lack of a four-way interaction $(F<1.00)$.

\section{Joystick Movement Scores}

Table 3 presents mean joystick movement scores plotted across five successive 30-frame segments for the Implicit, Rule-discovery, and Rule-application conditions. Reflecting the main effect of display segment $\left(F_{4,96}=11.76, p<\right.$ $.01, \eta^{2}=33$ ), all conditions produced maximum activity during the second and third segments of the pathways whereon a Glitch could appear. Although the pattern of means suggests differential activity for conditions, with the greatest activity in the early segments shown by the Rule-application condition and the least activity in early segments shown by the Rule-discovery condition, there was no significant interaction for Segment $\times$ Practice Condition $(F<1.00)$.

\section{Exit Questionnaire Findings}

The pattern of responses indicated that the Fade feature was a very salient feature regardless of practice condition as all but one participant (Rulediscovery condition) reported noticing the Fade feature. As this pattern suggests, there was no significant difference between conditions with respect to

Table 3. Mean joystick activity as a function of display segment and instructional condition

\begin{tabular}{lrrrrrl}
\hline Instructional Condition & \multicolumn{6}{c}{ Display Segment } \\
\cline { 2 - 7 } & 1 & 2 & 3 & 4 & 5 & Marginals \\
\hline Implicit & 6.12 & 7.58 & 7.45 & 5.27 & 3.70 & 6.02 \\
M & 6.30 & 5.39 & 4.42 & 3.02 & 2.08 & 4.20 \\
SD & & & & & & \\
Rule-discovery & 4.38 & 7.05 & 7.91 & 5.72 & 4.44 & 5.90 \\
M & 4.10 & 4.37 & 3.77 & 2.86 & 2.37 & 3.49 \\
SD & & & & & & \\
Rule-application & 6.55 & 8.54 & 8.01 & 5.14 & 3.97 & 6.44 \\
M & 7.12 & 5.83 & 3.92 & 2.49 & 2.35 & 4.34 \\
SD & & & & & & \\
Overall Marginals & 5.68 & 7.73 & 7.79 & 5.38 & 4.04 & \\
M & 6.05 & 5.27 & 4.05 & 2.81 & 2.29 & \\
SD & & & & &
\end{tabular}


reported perception of the presence of Fade feature in some of the pathways $\left[\chi^{2}(N=27)=2.27\right.$, ns]. In contrast, the pattern of responses showed that participants in the Rule-application (9 of 9) and the Rule-discovery (8 of 9) conditions more frequently noticed the presence of the Glitch feature than those in the Implicit condition (6 of 9). This nonsignificant pattern of data $\left[x^{2}(N=27)\right.$ $=4.91, \mathrm{~ns}]$ reflects the effect of explicit instructions, encouraging use or discovery of probability relationships, in drawing attention to important stimulus features. The effect of explicit instruction on noticing the predictive correlation between the Glitch and Fade features was not a significant observation across conditions $\left[\chi^{2}(N=27)=3.73, n s\right]$. However, participants in the Ruleapplication (5 of 9) and the Rule-discovery (7 of 9) conditions more frequently reported a correlation between the Glitch and Fade features than those in the Implicit ( 3 of 9) condition. Further, it should be noted that there was no significant difference between practice conditions with respect to noticing a correlation between the absence of a Glitch and the absence of a Fade feature ( 5 of 9 for each condition), and there was no significant difference between conditions with respect to perceived relationship between the presence or the absence of the Glitch and Fade features $(F s<1.00)$.

\section{Discussion}

As expected, the results clearly indicate that research participants, regardless of condition, improved performance with practice, as indicated by an overall pattern of reduced error across sessions. As predicted, participants used the probability information in performing the task regardless of their practice condition. The use of the probabilistic relationship between the Glitch and Fade features was convincingly demonstrated in each condition by a characteristic pattern of costs and benefits for trials involving both valid and invalid pathways. Although all conditions showed significant use of probability relationships between pathway features, clear evidence of the detrimental effect of providing explicit instructions was found, as predicted. Specifically, the explicit (Rule-application and Rule-discovery) conditions performed with greater error in catching the "ball," particularly during the early phases of practice, than the Implicit (noninstructed) condition. Further, in comparing the explicit conditions, the results indicated Rule-discovery led to worse performance than Rule-application. The pattern of greater error for the Rule-application than for the Implicit condition and the pattern of use of the probabilistic information for both the Ruleapplication and Implicit conditions replicate prior findings by Green and Flowers (1991), whereas the finding of the worst performance for the Rule-discovery condition is supportive of the prediction of even greater demands being placed on cognitive resources for this condition.

Differential performance between conditions, particularly during early practice sessions, strongly suggests fundamental differences between the per- 
formance consequences of implicit and explicit learning. It is believed that these differences in catching performance resulted from differential demands placed upon participants' cognitive resources which interfered with "proceduralization" of the motor aspects of the task for both the Rule-application condition and the Rule-discovery condition, with the greatest demands and costs occurring for the Rule-discovery condition. In other words, effort in trying to remember the rule and worrying about its application detracted from cognitive resources; however, trying to discover and apply the rule detracted even more from resources needed to perform optimally the fine-grained motor adjustments demanded by the catching task.

Support for the explanation based on limitations of cognitive resources can be seen when considering the apparent differences between conditions with respect to heightened attention to the Glitch feature as suggested by the finding from the exit questionnaire of more frequent noticing of the Glitch feature by participants in the explicit conditions. Obviously, less perception of the Glitch feature strongly suggests less opportunity for heightened attentional focus for the Implicit condition. The finding that only three of the participants in the Implicit condition noticed an association between the Glitch and Fade features reflects the relative lack of awareness and attention. Further, the pattern of joystick activity provides some support for the resource-limitation explanation. As first suggested by Green and Flowers (1991), in their discussion of differences between a noninstructed group and an explicitly instructed group engaged in a similar probability task, differences in joystick activity reflect the employment of different strategies (requiring different cognitive resources) by the noninstructed and explicitly instructed conditions. That is, the trend in our joystick activity scores suggests qualitatively different stylistic approaches associated with different cognitive demands. Specifically, the relatively lower activity of the Rule-discovery condition early in the pathway descent suggests a shift in attentional focus in discovery of the probability rules, whereas the relatively greater activity of the rule-application conditions suggests an attempt to become ready to apply forthcoming probability information.

An interesting parallel to and additional support for the resource-limitation explanation can be seen in Reber's interpretation (1989) of an earlier grammar learning study (Reber, 1976). Reber found that instructions about the existence of probability rules in a grammar learning situation produced lower performance, e.g., less rule learning, than noninstructed implicit learning (Reber, 1976). Reber suggested that explicit instructions produce a processing overload when subjects consciously try to discover rules. Although our task involved coordination of motor behavior with a continuously moving predictive target as opposed to prediction of such discrete events, a similar disruption involving increased cognitive demands seems to have occurred. Presumably the additional processing load and associated 
heightened attentional state resulting from subjects' awareness of and attempt to discovery rules results in the disruption of implicit learning processes which would otherwise allow effective use of sequential dependencies in these types of tasks.

The explanation involving cognitive demand and resource limitation has direct implications for instructional techniques in applied settings. In that attention-demanding visuomotor tasks appear particularly vulnerable to disruption by attempts to retain and apply verbally provided explicit probability information and by attempts to overtly direct resources for rulediscovery, it might be that simple noninstructed practice with general performance feedback as opposed to explicit instruction may lead to more efficient performance.

In addition to type of instruction, it is likely that disruption is also dependent on how much a particular task is resource-limited and the strength of the predictive relationships. Therefore, it is suggested that researchers investigate different types of tasks to discover which are most vulnerable to disruption by explicit instruction. Researchers should address the effects of manipulating the strength of probability relationships between events. It may be that higher predictability, e.g., greater than the $75 \%$, may offset costs of increased processing load and lead to more effective performance of explicitly instructed subjects. Further, in that the current findings showed that disruption resulting from explicit instructions occurred primarily in the early sessions, researchers should address the possibility that explicit instructional conditions may, with practice schedules longer than those in the current study, eventually enhance performance better than an implicit condition.

\section{References}

Abrams, M. (1987) Implicit learning in the psychiatrically impaired. Unpublished doctoral dissertation, City University of New York.

Berry, D. C., \& Broadbent, D. E. (1984) On the relationship between task performance and associated verbalizable knowledge. Quarterly Journal of Experimental Psychology: Human Experimental Psychology, 36A, 209-231.

Broadbent, D. E., \& Aston, B. (1978) Human control of a simulated economic system. Ergonomics, 21, 1035-1043.

Broadbent, D. E., FitzGerald, P., \& Broadbent, M. H. P. (1986) Implicit and explicit knowledge in the control of complex systems. British Journal of Psychology, 77, 33-.50.

Brooks, L. R. (1978) Nonanalytic concept formation and memory for instances. In E. Rosch \& B. B. Lloyd (Eds.), Cognition and categorization. New York: Wiley. Pp. 169-211.

Firth, C. D., \& Lang, R. J. (1979) Learning and reminiscence as a function of target predictability in a two-dimensional tracking task. Quarterly Journal of Experimental Psychology, 31, 103-109.

Flowers, J. H., Nelson, S. M., Carson, D., \& Larsen, L. (1984) Automatic and expectancybased priming effects in a digit naming task. Journal of Experimental Psychology: Human Perception and Performance, 10, 65-74. 
Flowers, J. H., Reed, D., \& Green, T. D. (1991) Priming by "predictive" context stimuli in visual classification. Bulletin of the Psychonomic Society, 29, 79-81.

Green, T. D., \& Flowers, J. H. (1991) Implicit versus explicit learning processes in a probabilistic continuous fine motor catching task. Journal of Motor Behavior, 23, 293-300.

Lewicki, P. (1986) Processing information about covariations that cannot be articulated. Journal of Experimental Psychology: Learning, Memory, and Cognition, 12, 135-146.

Lewicki, P., Czyzewska, M., \& Hoffman, H. (1987) Unconscious acquisition of complex procedural knowledge. Journal of Experimental Psychology: Learning, Memory, and Cognition, 13, 523-530.

Lewicki, P., Hill, T, \& Bizot, E. (1988) Acquisition of procedural knowledge about a pattern of stimuli that cannot be articulated. Cognitive Psychology, 20, 24-37.

Mather, J. A., \& Putchat, C. (1983) Parallel ocular and manual tracking responses to a continuously moving visual target. Journal of Motor Behavior, 15, 29-38.

Mathews, R. C., Buss, R. R., Stanley, W. B., Blanchard-Fields, F., Cho, J. R., \& Bruhan, B. (1989) Role of implicit and explicit processes in learning from examples: A synergistic effect. Journal of Experimental Psychology: Learning, Memory, and Cognition, 15, 1083-1100.

Millward, R. B. (1981) Models of concept formation. In R. E. Snow, P. A. Frederico, \& W. E. Montague (Eds.), Aptitude, learning, and instruction: Cognitive processes analysis. Hillsdale, NJ: Erlbaum. Pp. 245-295.

Millward, R. B., \& Reber, A. S. (1972) Probability learning: Contingent-event sequences with lags. American Journal of Psychology, 85, 81-98.

Neely, J. H. (1977) Semantic priming and retrieval from lexical memory: Roles of inhibitionless spreading activation and limited capacity attention. Journal of Experimental Psychology: General, 106, 226-254.

Nissen, M. J., \& Bullemer, P. (1987) Attentional requirements of learning: Evidence from performance measures. Cognitive Psychology, 19, 1-32.

Notterman, J. M., \& Tufano, D. R. (1980) Variables influencing outflow-inflow interpretations of tracking performance: Predictability of target motion, transfer function, and practice. Journal of Experimental Psychology: Human Perception and Performance, 6, 85-88.

Posner, M. I., \& Snyder, C. R. R. (1975) Attention and cognitive control. In R. L. Solso (Ed.), Information processing and cognition: The Loyola Symposium. Hillsdale, NJ: Erlbaum. Pp. 58-85.

Reber, A. S. (1966) A perceptual learning analysis of probability learning. Unpublished doctoral dissertation, Brown University, Providence, RI.

Reber, A. S. (1967) Implicit learning of artificial grammars. Journal of Verbal Learning and Verbal Behavior, 76, 317-327.

Reber, A. S. (1976) Implicit learning of synthetic languages: The role of instructional set. Journal of Experimental Psychology: Human Learning and Memory, 2, 88-94.

Reber, A. S. (1989) Implicit learning and tacit knowledge. Journal of Experimental Psychology: General, 118, 219-235.

Reber, A. S., \& Millward, R. B. (1968) Event observation in probability learning. Journal of Experimental Psychology, 77, 317-327.

Reber, A. S., \& Millward, R. B. (1971) Event tracking in probability learning. American Journal of Psychology, 84, 85-99.

Stadler, M. A. (1989) On learning complex procedural knowledge. Journal of Experimental Psychology: Learning, Memory, and Cognition, 15, 1061-1069.

Taylor, D. A. (1977) Time course of context effects. Journal of Experimental Psychology: General, 106, 404-426. 